\title{
Plasmoid instability in the semi-collisional regime
}

\author{
Pallavi Bhat ${ }^{1} \uparrow$ and Nuno F. Loureiro ${ }^{1}$ \\ ${ }^{1}$ Plasma Science and Fusion Center, Massachusetts Institute of Technology, Cambridge, MA 02139, USA
}

(Received 13 April 2018; revised 27 September 2018; accepted 28 September 2018)

We investigate analytically and numerically the semi-collisional regime of the plasmoid instability, defined by the inequality $\delta_{\mathrm{SP}} \gg \rho_{s} \gg \delta_{\text {in }}$, where $\delta_{\mathrm{SP}}$ is the width of a Sweet-Parker current sheet, $\rho_{s}$ is the ion sound Larmor radius and $\delta_{\text {in }}$ is the width of the boundary layer that arises in the plasmoid instability analysis. Theoretically, this regime is predicted to exist if the Lundquist number $S$ and the length of the current sheet $L$ are such that $\left(L / \rho_{s}\right)^{14 / 9}<S<\left(L / \rho_{S}\right)^{2}$ (for a sinusoidal-like magnetic configuration; for a Harris-type sheet the lower bound is replaced with $\left.\left(L / \rho_{s}\right)^{8 / 5}\right)$. These bounds are validated numerically by means of simulations using a reduced gyrokinetic model (Zocco \& Schekochihin, Phys. Plasmas, vol. 18 (10), 2011, 102309) conducted with the code Viriato. Importantly, this regime is conjectured to allow for plasmoid formation at relatively low, experimentally accessible, values of the Lundquist number. Our simulations obtain plasmoid instability at values of $S$ as low as $\sim 250$. The simulations do not prescribe a Sweet-Parker sheet; rather, one is formed self-consistently during the nonlinear evolution of the initial tearing mode configuration. This proves that this regime of the plasmoid instability is realizable, at least at the relatively low values of the Lundquist number that are accessible to current dedicated experiments.

Key words: astrophysical plasmas, plasma instabilities, plasma simulation

\section{Introduction}

Magnetic reconnection is a fundamental plasma physics phenomenon, relevant to laboratory, space and astrophysical systems (Biskamp 2000; Zweibel \& Yamada 2009; Uzdensky 2011). It involves a rapid topological rearrangement of the magnetic field, leading to efficient magnetic energy conversion and dissipation. Solar flares (Shibata \& Magara 2011) and sawtooth crashes in tokamaks (Hastie 1997) are two popular examples of processes where reconnection plays a key role; others include substorms in the Earth's magnetosphere (Dungey 1961; Burch et al. 2016), particle acceleration in jets and pulsar winds (Cerutti, Uzdensky \& Begelman 2012; Kagan et al. 2015; Werner et al. 2016), magnetized turbulence (e.g. Matthaeus \& Lamkin 1986; Servidio et al. 2009; Zhdankin et al. 2013; Cerri \& Califano 2017; Loureiro \& Boldyrev 2017; Mallet, Schekochihin \& Chandran 2017), etc.

$\dagger$ Email address for correspondence: pbhat@mit.edu 
In magnetohydrodynamic (MHD) plasmas, reconnection sites (current sheets) tend to be unstable to the formation of multiple small islands (or plasmoids) provided that the Lundquist number (defined as $S=L V_{A} / \eta$, where $L$ is the system size, $V_{A}$ the Alfvén speed and $\eta$ the magnetic diffusivity) is sufficiently large (typically, $S \gtrsim$ $10^{4}$ ). This is known as the plasmoid instability; the current sheets mediated by the plasmoids have an aspect ratio that is much smaller than that of the global sheet, thus triggering fast reconnection (Loureiro, Schekochihin \& Cowley 2007; Lapenta 2008; Bhattacharjee et al. 2009; Cassak, Shay \& Drake 2009; Samtaney et al. 2009; Huang \& Bhattacharjee 2010; Uzdensky, Loureiro \& Schekochihin 2010; Loureiro et al. 2012; Loureiro, Schekochihin \& Uzdensky 2013; Loureiro \& Uzdensky 2016).

In weakly collisional plasmas, where the frozen-flux constraint is broken by kinetic effects instead of collisions, plasmoids are also abundantly observed (e.g. Drake et al. 2006; Ji \& Daughton 2011; Daughton \& Roytershteyn 2012), suggesting that plasmoid generation and dynamics are robust and fundamental features of reconnecting systems, regardless of the collisionality of the ambient plasma.

Because of its perceived importance - from determining the reconnection rate in MHD plasmas to its possible role in the reconnection onset (Pucci \& Velli 2014; Comisso et al. 2016; Uzdensky \& Loureiro 2016; Tolman, Loureiro \& Uzdensky 2018) and in the energy partition (Loureiro et al. 2012; Numata \& Loureiro 2015) and particle acceleration and plasma heating (Drake et al. 2006; Giannios, Uzdensky \& Begelman 2009; Oka et al. 2010; Cerutti et al. 2013; Sironi \& Spitkovsky 2014; Guo et al. 2015; Zhou et al. 2015; Sharma, Mitra \& Oberoi 2017; Werner \& Uzdensky 2017 ) - the plasmoid instability has been the subject of a multitude of theoretical and numerical studies (see Loureiro \& Uzdensky (2016) for a brief review). There are also abundant reports of plasmoid observation in solar flares (Milligan et al. 2010; Nishizuka et al. 2010; Liu, Chen \& Petrosian 2013), coronal jets (Zhang \& Ji 2014) and in the Earth's magnetotail (Moldwin \& Hughes 1992; Zong et al. 2004) and in the magnetospheres of other planets (Jackman, Slavin \& Cowley 2011; Zhang et al. 2012; DiBraccio et al. 2015). This, however, contrasts starkly with plasmoid detection and investigation in laboratory experiments, which have so far been relatively limited, with only a handful of studies reporting plasmoid observation (Fox, Bhattacharjee \& Germaschewski 2012; Dorfman et al. 2013; Jara-Almonte et al. 2016; Olson et al. 2016; Hare et al. 2017a,b, 2018). In all cases, these observations have occurred in non-MHD regions of parameter space (dedicated reconnection experiments have not been able to reach $S>10^{4}$, although future ones might (Forest et al. 2015; Ji et al. 2015)), and lack a solid theoretical footing.

In a recent paper, Loureiro \& Uzdensky (2016) have identified a plasma collisionality regime where the requirement for triggering the plasmoid instability is significantly eased with respect to its pure MHD counterpart. In essence, this regime relies on collisionality being high enough that an MHD current sheet may form in the first place (i.e. the current sheet thickness exceeds any kinetic scale); but small enough that when such a sheet is analysed for its stability to plasmoid formation, two-fluid effects can no longer be neglected. Interestingly, some of the above mentioned experimental reports of plasmoid detection (Dorfman et al. 2013; Jara-Almonte et al. 2016; Hare et al. $2017 a, b, 2018)$ seem to sit in, or very close to, this region of parameter space (Hare 2017), and it is conceivable that they provide experimental evidence of the existence of this novel regime.

The aim of this paper is to report a set of numerical experiments designed to confirm the existence of the semi-collisional plasmoid instability, with particular focus on experimentally accessible values of the Lundquist number, and precisely map out the regions of parameter space inhabited by it. 


\section{The semi-collisional plasmoid instability}

The linear theory of the plasmoid instability in MHD plasmas (Loureiro et al. 2007; Bhattacharjee et al. 2009; Loureiro et al. 2013) assumes the existence of a Sweet-Parker (SP) current sheet (Parker 1957; Sweet 1958), which is taken as the background equilibrium whose stability is analysed. In standard tearing mode fashion (Furth, Killeen \& Rosenbluth 1963), the calculation divides the spatial domain into an outer region, where resistivity effects can be ignored, and an inner region - a boundary layer of thickness $\delta_{\text {in }}$ - where resistivity matters.

Let us revisit this question adding minimal kinetic two-fluid effects: we wish to consider the case where the ion sound Larmor radius, $\rho_{s}$, although smaller than the thickness of the SP current sheet, $\delta_{\mathrm{SP}}$, is however larger than the boundary layer of the MHD linear plasmoid instability: $\delta_{\mathrm{SP}} \gg \rho_{s} \gg \delta_{\text {in }} \cdot{ }^{1}$ This obviously implies that MHD is no longer a sufficient description. On the other hand, collisionality $\left(v_{\mathrm{ei}}\right)$ will still be taken to be large enough that the frozen-flux constraint is broken by resistivity, instead of electron inertia; i.e. $\delta_{\text {in }} \gg d_{e}$, with $d_{e}$ the electron skin depth (which results from the ordering $v_{\mathrm{ei}} \gg \gamma$, where $\gamma$ is the tearing mode growth rate). Nonetheless, the semi-collisional tearing mode requires $\delta_{\text {in }} \ll \rho_{s}$ and transitions to the usual collisional (MHD) tearing mode in the limit $\delta_{\text {in }} \gg \rho_{s}$, and becomes the collisionless tearing mode when the collision frequency is decreased such that $v_{\mathrm{ei}} \ll \gamma$ (Drake \& Lee 1977). On a qualitative level, this regime does not require ion finite Larmor orbit effects (i.e. it exists in the limit $\rho_{i} \rightarrow 0$ as long as $\rho_{s}$ remains finite). But our arguments below assume $\rho_{i} \sim \rho_{s}$, and our simulations will consider the case when $T_{0 i}=T_{0 e}$.

The expressions for the growth rate of the plasmoid instability in this regime can be obtained from the appropriate tearing mode theory (Drake \& Lee 1977; Pegoraro \& Schep 1986; Zocco \& Schekochihin 2011), following the usual procedure of replacing the equilibrium scale length with $\delta_{\mathrm{SP}} \sim L S^{-1 / 2}$, where $L$ is the length of the current sheet (Tajima \& Shibata 2002; Bhattacharjee et al. 2009).

For small values of the tearing mode instability parameter $\Delta^{\prime}$, i.e. $\Delta^{\prime} \delta_{\text {in }} \ll 1$, we find

$$
\begin{gathered}
\gamma L / V_{A} \sim(k L)^{2 / 3}\left(\Delta^{\prime} \rho_{s}\right)^{2 / 3}, \\
\delta_{\text {in }} / L \sim\left(\Delta^{\prime} \rho_{S}\right)^{1 / 6}(k L)^{-1 / 3} S^{-1 / 2},
\end{gathered}
$$

where $\gamma$ is the growth rate of a mode with wavenumber $k$. This expression can be simplified if $\Delta^{\prime}$ is not too small, such that it can be approximated as $\Delta^{\prime} \delta_{\mathrm{SP}} \sim 1 /\left(k \delta_{\mathrm{SP}}\right)$, as pertains to the usual Harris-like magnetic configuration (Harris 1962). In that case, we obtain

$$
\gamma L / V_{A} \sim\left(\rho_{s} / L\right)^{2 / 3} S^{2 / 3}
$$

and the validity condition $\Delta^{\prime} \delta_{\text {in }} \ll 1$ becomes $k L \gg\left(\rho_{s} / L\right)^{1 / 9} S^{4 / 9}$. Note that this expression is independent of $k$ to lowest order.

In the opposite limit of large $\Delta^{\prime}$, i.e. $\Delta^{\prime} \delta_{\text {in }} \gg 1$, we instead have

$$
\begin{aligned}
& \gamma L / V_{A} \sim\left(\rho_{s} / L\right)^{4 / 7} S^{2 / 7}(k L)^{6 / 7}, \\
& \delta_{\text {in }} / L \sim\left(\rho_{s} / L\right)^{1 / 7}(k L)^{-2 / 7} S^{-3 / 7}
\end{aligned}
$$

\footnotetext{
${ }^{1}$ An additional requirement is that the electron skin depth, $d_{e}=c / \omega_{\text {pe, }}$ with $\omega_{\text {pe }}$ the electron plasma frequency, is negligible, i.e. $\delta_{\text {in }} \gg d_{e}$. A further generalization of the theory to include electron inertia effects is possible - see Loureiro \& Uzdensky (2016).
} 
The fastest growing mode is yielded by the intersection of these two branches (Loureiro \& Uzdensky 2016) ${ }^{2}$

$$
\begin{gathered}
\gamma_{\max } L / V_{A} \sim\left(\rho_{s} / L\right)^{2 / 3} S^{2 / 3}, \\
k_{\max } L \sim\left(\rho_{s} / L\right)^{1 / 9} S^{4 / 9}, \\
\delta_{\text {in }} / L \sim\left(\rho_{s} / L\right)^{1 / 9} S^{-5 / 9}
\end{gathered}
$$

The validity of these expressions rests on two conditions: $\delta_{\mathrm{SP}} \gg \rho_{s}$, and $\rho_{s} \gg \delta_{\text {in }}$. Using (2.8), these therefore imply that the semi-collisional plasmoid instability inhabits the region of parameter space defined by ${ }^{3}$

$$
\left(L / \rho_{s}\right)^{2} \gg S \gg\left(L / \rho_{s}\right)^{8 / 5} .
$$

An alternative current sheet profile worth considering - and the one we will make use of in this paper - is that of a sinusoidal-like magnetic field, $B_{y}(x)=B_{0} \sin (x / a)$, for which $\Delta^{\prime} \delta_{\mathrm{SP}} \sim 1 /\left(k \delta_{\mathrm{SP}}\right)^{2}$. For small $\Delta^{\prime}$, we obtain,

$$
\begin{gathered}
\gamma L / V_{A} \sim\left(\rho_{s} / L\right)^{2 / 3}(k L)^{-2 / 3} S, \\
\delta_{\text {in }} \sim\left(\rho_{s} / L\right)^{1 / 6}(k L)^{-2 / 3} S^{-1 / 4},
\end{gathered}
$$

valid if $k L \gg\left(\rho_{s} / L\right)^{1 / 16} S^{15 / 32}$. For large $\Delta^{\prime}$, the scaling for $\gamma L / V_{A}$ is same as in (2.4), as there is no explicit dependence on $\Delta^{\prime}$. The fastest growing mode is therefore characterized by

$$
\begin{aligned}
\gamma_{\max } L / V_{A} & \sim\left(\rho_{s} / L\right)^{5 / 8} S^{11 / 16}, \\
k_{\max } L & \sim\left(\rho_{s} / L\right)^{1 / 16} S^{15 / 32}, \\
\delta_{\text {in }} / L & \sim\left(\rho_{s} / L\right)^{1 / 8} S^{-9 / 16} .
\end{aligned}
$$

Figure 1 illustrates both limits of the dispersion relation, and their intersection, for two different combinations of the two relevant parameters, $S$ and $L / \rho_{s}$. In the Appendix we recover these scalings via direct numerical simulation, confirming both their validity and the ability of the code Viriato (Loureiro et al. 2016), which we will employ in this paper (see $\S \S 3$ and 4 ), to recover them.

In this case of a sinusoidal-like current sheet profile, equation (2.9) is replaced by:

$$
\left(L / \rho_{s}\right)^{2} \gg S \gg\left(L / \rho_{s}\right)^{14 / 9} .
$$

The lower bound here has only a slightly smaller exponent than (and is in practice difficult to discern from) the Harris-like case of (2.9).

Equations (2.9) and (2.15) lead to the interesting suggestion that this particular version of the plasmoid instability can be obtained at relatively low values of $S$, provided that the system (the current sheet length $L$, to be precise) is not too large compared with $\rho_{s}$. In other words, the lower bound of $S \sim 10^{4}$ that pertains to the MHD version of the plasmoid instability (Biskamp 1986; Loureiro et al. 2005; Samtaney et al. 2009; Baty 2014) is replaced by a function in the semi-collisional regime, $\left(L / \rho_{s}\right)^{14 / 9}$, or $\left(L / \rho_{s}\right)^{8 / 5}$, as appropriate. This growth of plasmoids at low $S$ is possible in the semi-collisional regime due to the presence of the $\rho_{s}$ scale, which is unavailable in MHD. The tearing calculation in this regime has to account for

\footnotetext{
${ }^{2}$ Note that the scaling for $\delta_{\text {in }}$, equation (2.8), has been corrected from Loureiro \& Uzdensky (2016).

${ }^{3}$ In addition, the existence of the plasmoid instability (irrespective of the collisionality regime) requires that $\gamma L / V_{A} \gg 1$ and $k L \gg 1$; both of these conditions yield requirements on $S$ and $L / \rho_{S}$ that are less demanding than the rightmost inequality in $(2.9)$, so $\left(L / \rho_{S}\right)^{8 / 5}$ should be the correct lower bound.
} 


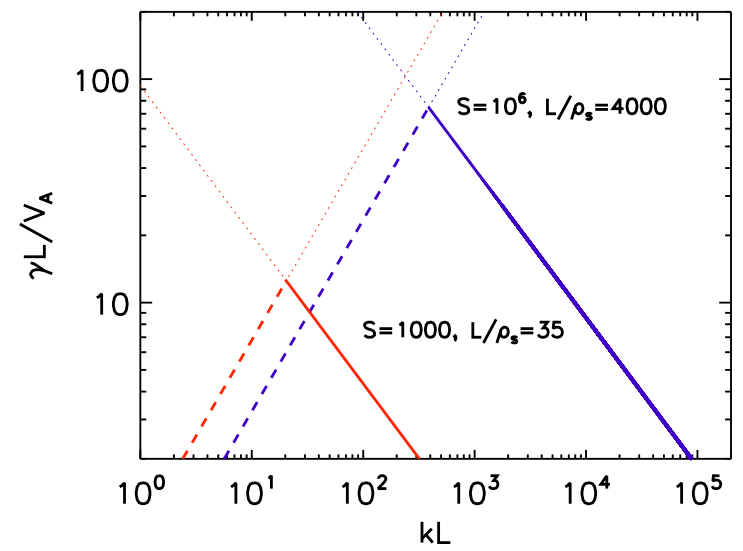

FIGURE 1. Dispersion relation for the semi-collisional plasmoid instability for two different combinations of the relevant parameters: $\left(S, L / \rho_{S}\right)=(1000,35)$ (red lines) and $\left(S, L / \rho_{s}\right)=\left(10^{6}, 4000\right)$ (blue lines). Solid lines represent the case of $\Delta^{\prime} \delta_{\text {in }} \ll 1(2.11)$, whereas dashed lines are for the case of $\Delta^{\prime} \delta_{\text {in }} \gg 1$ (2.4). Respectively, these lines are only valid to the right, or to the left, of their intersection. Their point of intersection provides an estimate of the most unstable wavenumber and corresponding growth rate.

nested boundary layers instead of a single one: this introduces an extra parameter in the problem and makes the critical Lundquist number (derived from the requirement that the condition for semi-collisional regime, $\delta_{\mathrm{SP}}>\rho_{s}>\delta_{\text {in }}$ be satisfied) become dependent on this new parameter.

From both the experimental and the numerical points of view, this feature of low critical Lundquist number is a significant advantage. In particular, this regime should be available to reconnection experiments such as the magnetic reconnection experiment (MRX) (Yamada et al. 1997), terrestrial reconnection experiment (TREX) (Olson et al. 2016), facility for laboratory reconnection experiments (FLARE) (Ji \& Daughton 2011) and the mega ampere generator for plasma implosion experiments (Magpie) (Hare et al. 2017a,b, 2018). Indeed, as we mention above, it is tempting to attribute recent reports of experimental plasmoid observation (Dorfman et al. 2013; Jara-Almonte et al. 2016; Hare et al. 2017a,b, 2018) to this version of the plasmoid instability - or to its $\beta \sim 1$ analogue (Baalrud et al. 2011) ( $\beta$ is the ratio of the plasma pressure to the magnetic pressure). In the weak guide field or $\beta \sim 1$ case, the relevant scale is the ion-inertial scale $d_{i}$ (instead of $\rho_{s}$ ), and the same feature of scale-dependent critical Lundquist number is expected to hold.

Despite these speculations and conjectures, the existence of this regime of the plasmoid instability has not been confirmed via direct numerical simulations, and indeed there are a couple of issues that may raise suspicion. In particular, we note that all of the scalings above are predicated on there being an asymptotic separation between the scales involved, namely $\delta_{\mathrm{SP}} \gg \rho_{s} \gg \delta_{\text {in. }}{ }^{4}$ As the Lundquist number is

\footnotetext{
${ }^{4}$ Note that the condition for semi-collisional regime should not be confused with a similar looking criterion specified in a scenario of hierarchy of plasmoids and interplasmoid current sheets, namely, $\delta_{\mathrm{SP}}(L)>\rho_{S}>\delta_{c}$ (see Uzdensky et al. 2010). Here, $\delta_{\mathrm{SP}}(L)$ refers to the width of the primary global SP current sheet and $\delta_{c}$ is the width of the smallest interplasmoid SP current sheet which is marginally unstable to the plasmoid instability. This criterion guarantees a transition of the plasmoid hierarchy from MHD to kinetic scales.

The scalings (2.8) and (2.14) allow us to make this criterion more precise. Consider the plasmoid cascade. For an interplasmoid current sheet with the width $\delta^{(N)}\left(\delta^{(2)}, \delta^{(3)}\right.$ are the secondary and tertiary SP current sheets,
} 
made smaller, this scale separation is inevitably lost, and thus the claim that the semi-collisional plasmoid instability may be obtainable at the relatively low values of $S$ and $L / \rho_{s}$ that are within the reach of existing experiments needs careful numerical validation.

An additional concern of significant relevance is that of whether the Sweet-Parker sheet that is assumed as the background for the instability derived here is realizable. As has been pointed out (Loureiro et al. 2007; Pucci \& Velli 2014; Uzdensky \& Loureiro 2016), the existence of a super-Alfvénic instability whose growth rate diverges as $S \rightarrow \infty$ indicates that the equilibrium that it arises from, may never form in the first place. We think this claim is pertinent at high values of the Lundquist number. However, in the opposite limit of relatively low $S$ with which we are mostly concerned here, where the instability is only mildly super-Alfvénic, a Sweet-Parker sheet may still form and beget the instability.

This paper aims to answer these questions by means of direct numerical simulations of a reconnecting system where the current sheet is not prescribed, but rather allowed to form self-consistently.

\section{The model}

The weak collisionality of a large number of reconnecting environments demands the use of a kinetic description. In the most general case, one is forced to adopt a first-principles formalism (such as particle-in-cell, or the six-dimensional (6-D) Vlasov (or Boltzmann) equation), with its inherent analytical and numerical complexity. In many situations, however, a strong component of the magnetic field is present that is perpendicular to the reconnection plane. This guide field offers an opportunity for significant simplification: the 5-D gyrokinetic formalism (Frieman \& Chen 1982; Howes et al. 2006).

Further simplification is possible if, in addition, one considers plasmas such that the electron $\beta$ is sufficiently low to be comparable to the electron-to-ion mass ratio $\left(m_{e} / m_{i}\right)$; a case in point is the solar corona, as mentioned earlier in $\S 2$. Leveraging on these assumptions (strong guide field and low $\beta$ ), a reduced-gyrokinetic formalism was recently derived (dubbed the 'kinetic reduced electron heating model', or KREHM) (Zocco \& Schekochihin 2011). One of its appealing features is that the phase space is reduced further to four dimensions (position vector and velocity in the guide-field direction only), thus rendering computations, and even analytic theory, significantly more manageable than fully kinetic approaches.

In this work, we use the KREHM equations to investigate the plasmoid instability in the semi-collisional regime. We will restrict our numerical investigations to the two spatial dimensions comprising the reconnection plane, $(x, y)$. With this restriction, the KREHM equations become:

$$
\frac{1}{n_{0 e}} \frac{\mathrm{d} \delta n_{e}}{\mathrm{~d} t}=\frac{1}{B_{0}}\left\{A_{\|}, \frac{e}{c m_{e}} d_{e}^{2} \nabla_{\perp}^{2} A_{\|}\right\}
$$

and so on), if the corresponding inner layer $\delta_{\text {in }}^{(N)}$ is larger than $\rho_{s}$, then the arising plasmoids are still in MHD regime. However, if at any point in the plasmoid cascade one obtains $\rho_{S}>\delta_{\text {in }}^{(N)}$, then the system transitions into the semi-collisional regime. Using the relationship $\delta_{c} \sim L_{c} S_{c}^{-1 / 2}$, where $S_{c} \sim 10^{4}$ is the critical value of the Lundquist number to obtain the plasmoid instability in MHD, it is easy to conclude that a transition to the semi-collisional regime must occur at some point in the plasmoid hierarchical cascade if $S>S_{C}^{3 / 8}\left(L / \rho_{S}\right)$, for a Harris-type sheet, or $S>S_{c}^{5 / 14}\left(L / \rho_{S}\right)$, for a sinusoidal-type sheet. 


$$
\begin{gathered}
\frac{\mathrm{d}}{\mathrm{d} t}\left(A_{\|}-d_{e}^{2} \nabla_{\perp}^{2} A_{\|}\right)=\eta \nabla_{\perp}^{2} A_{\|}-\frac{c T_{0 e}}{e B_{0}}\left\{A_{\|},\left(\frac{\delta n_{e}}{n_{0 e}}+\frac{\delta T_{\| e}}{T_{0 e}}\right)\right\} \\
\frac{\mathrm{d} g_{e}}{\mathrm{~d} t}-\frac{v_{\|}}{B_{0}}\left\{A_{\|},\left(g_{e}-\frac{\delta T_{\| e}}{T_{0 e}} F_{0 e}\right)\right\}=C\left[g_{e}\right]-\left(1-\frac{2 v_{\|}^{2}}{v_{\text {the }}^{2}}\right) \frac{F_{0 e}}{B_{0}}\left\{A_{\|}, \frac{e}{c m_{e}} d_{e}^{2} \nabla_{\perp}^{2} A_{\|}\right\},
\end{gathered}
$$

where

$$
\frac{\mathrm{d}}{\mathrm{d} t}=\frac{\partial}{\partial t}+\frac{c}{B_{0}}\{\phi, \ldots\} .
$$

Here, $\phi$ is electrostatic potential and $\{\ldots\}$ denotes the Poisson bracket, defined for any two fields $P, Q$ as $\{P, Q\}=\partial_{x} P \partial_{y} Q-\partial_{y} P \partial_{x} Q$.

Equations (3.1) and (3.2) evolve the zeroth and first moments of the perturbed electron distribution function, $\delta f_{e}$. The zeroth moment is the electron density perturbation, $\delta n_{e}$. The first moment is the parallel electron flow, $u_{\| e}$, and is related to the parallel component of the magnetic vector potential $A_{\|}$by $u_{\| e}=\left(e / c m_{e}\right) d_{e}^{2} \nabla_{\perp}^{2} A_{\|}$. The perturbed electron distribution function is given by

$$
\delta f_{e}=g_{e}+\left(\delta n_{e} / n_{0 e}+2 v_{\|} u_{\| e} / v_{\text {the }}^{2}\right) F_{0 e},
$$

where $F_{0 e}=n_{0 e} /\left(\pi / v_{\text {the }}^{2}\right)^{3 / 2} \exp \left[-\left(v_{\|}^{2}+v_{\perp}^{2}\right) / v_{\text {the }}^{2}\right]$ is the Maxwellian equilibrium, and $v_{\|}$and $v_{\perp}$ are, respectively, the velocity coordinates parallel and perpendicular to the guide-field direction. The electron thermal speed is $v_{\text {the }}=\sqrt{2 T_{0 e} / m_{e}}$, with $e$ the electron charge, and $m_{e}$ its mass. Note that this is a $\delta f$ formulation, so any fluctuating quantity is necessarily much smaller than the equilibrium quantity.

The quantity $g_{e}$ is dubbed the reduced electron distribution function; its evolution is given by (3.3). It contains all the moments of $\delta f_{e}$ higher than $\delta n_{e}$ and $u_{\| e}$. For example, parallel temperature fluctuations (second-order moment) are given by $\delta T_{\| e} / T_{0 e}=\left(1 / n_{0 e}\right) \int \mathrm{d}^{3} \boldsymbol{v}\left(2 v_{\|}^{2} / v_{\text {the }}^{2}\right) g_{e}$. On the right-hand side, $C\left[g_{e}\right]$ denotes the collision operator, and the second term represents what survives of the so-called 'gyrokinetic potential' in this expansion.

The background magnetic guide field is $B_{0}$; and $n_{0 e}, T_{0 e}$ are the background electron density and temperature, respectively. Other symbols introduced above are the electron skin depth, $d_{e}=c / \omega_{\mathrm{pe}}$, with $\omega_{\mathrm{pe}}=\sqrt{4 \pi n_{0 e} \mathrm{e}^{2} / m_{e}}$ the electron plasma frequency.

The perturbed electron density, $\delta n_{e}$, and the electrostatic potential are related via the gyrokinetic Poisson law,

$$
\frac{\delta n_{e}}{n_{0 e}}=\frac{1}{\tau}\left(\hat{\Gamma}_{0}-1\right) \frac{\mathrm{e} \phi}{T_{0 e}},
$$

where, $\tau=T_{0 i} / T_{0 e}$ is the ion to electron background temperature ratio, and $\hat{\Gamma}_{0}$ is a real space operator whose Fourier transform is $\Gamma_{0}(\alpha)=I_{0}(\alpha) \mathrm{e}^{-\alpha}$, with $I_{0}$ the modified Bessel function of zeroth order and $\alpha=k_{\perp}^{2} \rho_{i}^{2} / 2$, with $\rho_{i}=v_{\text {thi }} / \Omega_{i}$ the ion Larmor radius, $v_{\text {thi }}=\sqrt{2 T_{0 i} / m_{i}}$ the ion thermal velocity and $\Omega_{i}=|\mathrm{e}| B_{0} / m_{i} c$ the ion gyro frequency.

To make contact with a more familiar set of equations, note that, in the fluid limit $\left(d_{e}, \rho_{i}, \rho_{s} \rightarrow 0\right)$, equations (3.1) and (3.2) decouple from (3.3) and reduce to the momentum and induction equations of reduced MHD. However, when kinetic effects are retained, Ohm's law couples to the kinetic equation (3.3) via parallel electron temperature fluctuations.

Observe that (3.3) does not explicitly depend on $v_{\perp}$. Provided that one chooses a collision operator which is itself also independent of $v_{\perp}$, this coordinate can be 
integrated out, yielding a reduced electron distribution function that is effectively fourdimensional only, $g_{e}=g_{e}\left(x, y, z, v_{\|}, t\right)$. One model collision operator that exhibits this property is the Lenard-Bernstein operator (Lenard \& Bernstein 1958), modified to conserve the pertinent quantities (Zocco \& Schekochihin 2011). Then (3.3) can be more conveniently solved in terms of its expansion in Hermite polynomials:

$$
g_{e}\left(x, y, z, v_{\|}, t\right)=\sum_{m=2}^{\infty} \frac{1}{2^{m} m !} H_{m}\left(v_{\|} / v_{\text {the }}\right) g_{m}(x, y, z, t) F_{0 e}\left(v_{\|}\right),
$$

where $H_{m}$ denotes the Hermite polynomial of order $m$ and $g_{m}$ is its coefficient. Inserting this expansion into (3.3) yields a coupled set of fluid-like equations for the coefficients of the Hermite polynomials, where by construction $g_{0}=g_{1}=0$, and for $m \geqslant 2$ we have

$$
\begin{gathered}
\frac{\mathrm{d} g_{m}}{\mathrm{~d} t}-\frac{v_{\text {the }}}{B_{0}}\left(\sqrt{\frac{m+1}{2}}\left\{A_{\|}, g_{m+1}\right\}+\sqrt{\frac{m}{2}}\left\{A_{\|}, g_{m-1}\right\}\right) \\
=\frac{\sqrt{2} \delta_{m, 2}}{B_{0}}\left\{A_{\|}, \frac{e}{c m_{e}} d_{e}^{2} \nabla_{\perp}^{2} A_{\|}\right\}-v_{\text {ei }}\left(m g_{m}-2 \delta_{m, 2} g_{2}\right),
\end{gathered}
$$

where $v_{\mathrm{ei}}$ is the electron-ion collision frequency, $v_{\mathrm{ei}}=\eta / d_{e}^{2}$. The kinetic equations solved by means of Hermite expansion requires a closure. A way to close the set of equations is to demand that at some $m=M$, the collision term becomes significant such that $g_{M+1} / g_{M} \ll 1$. This constraint will truncate the kinetic equations at $g_{M}$ as $g_{M+1}$ can be written in terms of $g_{M}$ (Zocco \& Schekochihin 2011; Zocco et al. 2015; Loureiro et al. 2016; White \& Hazeltine 2017). This type of closure also recovers the semi-collisional limit exactly.

\section{Numerical set-up}

Equations (3.1), (3.2), (3.6) and (3.8) are solved numerically on a two-dimensional grid of size $L_{x} \times L_{y}$, using the pseudo-spectral code Viriato (Loureiro et al. 2016). Periodic boundary conditions are employed in both directions. The numerical configuration is akin to that employed in Loureiro et al. (2005) and, as we will show, is such that one can self-consistently obtain an SP current sheet whose stability to plasmoid formation can then be studied. Specifically, the input parameters can be specified in a way as to lead to the dynamic formation of a SP current sheet that meets the conditions of the semi-collisional regime that we have discussed earlier.

The initial equilibrium is $A_{\|}(x, y, t=0)=A_{\| 0} / \cosh ^{2}(x)$, where $A_{\| 0}=3 \sqrt{3} / 4$, such that the maximum of the reconnecting field, $B_{y}=\mathrm{d} A_{\|} / \mathrm{d} x$, is $B_{y, \max }=1$. This equilibrium is destabilized with a small amplitude (linear) perturbation which seeds the fastest growing tearing mode; in all simulations, this is the longest wavelength mode that fits in the simulation box. Once in the nonlinear stage, the tearing mode undergoes $X$-point collapse (Waelbroeck 1989; Loureiro et al. 2005), and a current sheet forms which is consistent with the SP scaling (as we shall confirm). The plasmoid instability is then triggered, or not, depending on the values of $S$ and $\rho_{s}$ specified in the simulation.

The length of the SP current sheet can be varied by changing the instability parameter, $\Delta^{\prime}(k)$, pertaining to the initial tearing mode (Loureiro et al. 2005). In practice, this is achieved by changing the length of the box in the outflow direction, $L_{y}$. 
The resolution of a simulation in the inflow $(x)$ direction is set by the size of the inner boundary layer that is expected to arise due to the plasmoid instability, estimated using (2.8). The resolution demands in the outflow $(y)$ direction are less stringent, and are determined on a ad hoc basis.

An additional constraint on our runs is that the electron inertia play no role. This is insured by setting it to be smaller than the resolution for any given simulation. Therefore, in all runs, the frozen-flux constraint is broken by resistivity. Note that no hyper-resistivity (or hyper-viscosity) is used in these runs, ensuring that the actual Lundquist number in the simulations is determined by the resistivity that we specify. The simulations also employ a finite viscosity, set equal to the magnetic diffusivity.

A final choice has to do with the number of Hermite polynomials to keep in the simulations. For all runs reported here, the highest-order polynomial is $M=4-$ this ought to be sufficient given the relatively high collisionality of our simulations (and indeed we find that in all our runs resistivity is the dominant energy dissipation channel). To check convergence, we performed one test using instead $M=10$, and observed that in the spectrum of $\left|g_{m}\right|^{2} / 2$, the energy at $m>3-4$, is lower than that at $m=2$ by orders of magnitude, indicating that the power transferred to the Hermite polynomials of higher order is not significant. In all runs, the convergence of the Hermite representation is accelerated by the use of a hyper-collision operator (see Loureiro et al. (2016) for details).

\section{Results}

As previously stated, our main aim is to numerically ascertain the existence of the semi-collisional plasmoid instability and validate the bounds of the parameter space defined by $S$ and $L / \rho_{s}$ where this instability is expected to be active. We specifically wish to focus on the instability's existence at modest, experimentally accessible, values of the Lundquist number. To this effect, we perform a series of runs as listed in table 1. Amongst other parameters, the table lists the length of the current sheet, $L$, that is dynamically obtained during the nonlinear evolution of the primary tearing mode (which results from the collapse of the $X$-point, as previously described). This length is measured using a full width at half-maximum estimate ${ }^{5}$ (as is the current sheet thickness, $\delta$ ) just before the plasmoid appears, and it is this length that is used to estimate the Lundquist number that is also listed in table 1 (the magnitude of the upstream magnetic field remains unchanged by the $X$-point collapse).

The first step in the description of our results is the characterization of the current sheet that is dynamically obtained from the $X$-point collapse of the primary tearing mode. The theory of the semi-collisional plasmoid instability laid out in $\S 2$ assumes a SP sheet as the background equilibrium; and so it is important to determine whether indeed that is the case in our simulations. In figure 2 , we plot $\delta / L$ as a function of the Lundquist number $S$, obtained from all the runs. We find, as shown in figure 2 by the blue stars (Runs A to H) and red diamonds (Runs I, J, K), that the current sheets in these runs follow the SP scaling. The system in these runs is initially purely in the MHD regime as the inner boundary layer thickness of the primary tearing mode is larger than the kinetic scales. And thus, upon $X$-point collapse of the MHD tearing mode, the current sheets that form are expected to follow the SP scaling (Loureiro et al. 2005), which indeed bears out.

\footnotetext{
${ }^{5}$ This is different from Ji \& Daughton (2011), where $L$ represents the half-length of the reconnecting sheet. This difference amounts to a shift equal to $\log (2)$ between our reconnection phase diagram and theirs.
} 


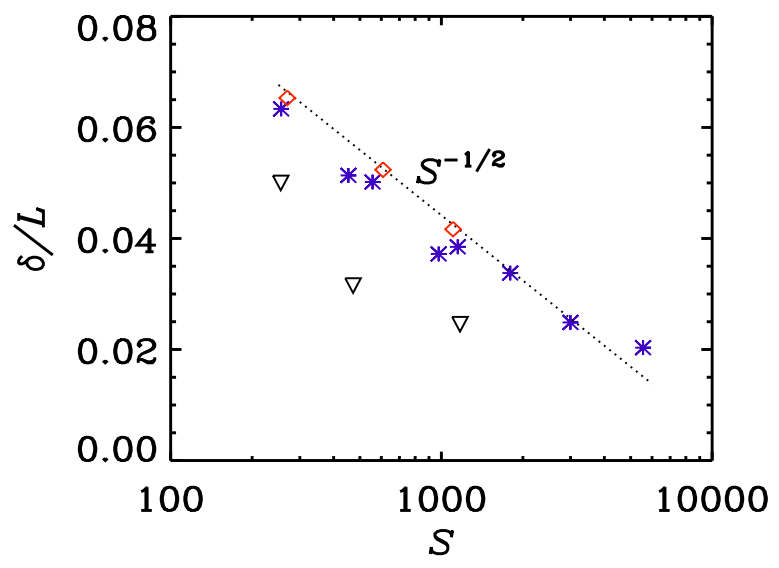

FIGURE 2. The ratio of current sheet width $\delta$ to its length $L$ against the Lundquist number $S$ is shown for Runs A through $\mathrm{H}$ (blue stars), I, J and K (red diamonds), and $\mathrm{L}, \mathrm{M}$ and $\mathrm{N}$ (black inverted triangles). Refer to table 1 for details of these runs. The dotted line indicates an $S^{-1 / 2}$ slope, expected for Sweet-Parker current sheets.

$\begin{array}{lcccccccccc}\text { Run } & \rho_{S} & L & L / \rho_{s} & S & \delta_{\text {in }} & N_{x} \times N_{y} & L_{y} / 2 \pi & \eta & \delta_{\mathrm{SP}}>\rho_{s} ? & \rho_{s}>\delta_{\text {in }} ? \\ \text { A } & 0.12 & 3.01 & 25.1 & 256 & 0.089 & 512 \times 128 & 1.75 & 0.012 & \text { Yes } & \text { Yes } \\ \text { B } & 0.1 & 2.73 & 27.3 & 453 & 0.058 & 1024 \times 256 & 1.695 & 0.00657 & \text { Yes } & \text { Yes } \\ \text { C } & 0.07 & 2.91 & 41.6 & 557 & 0.052 & 512 \times 128 & 1.678 & 0.00583 & \text { Yes } & \text { Yes } \\ \text { D } & 0.06 & 2.58 & 43.0 & 977 & 0.033 & 1024 \times 256 & 1.678 & 0.003 & \text { Yes } & \text { Yes } \\ \text { E } & 0.036 & 2.52 & 70.0 & 1149 & 0.028 & 1024 \times 256 & 1.587 & 0.0025 & \text { Yes } & \text { Yes } \\ \text { F } & 0.04 & 2.28 & 57.0 & 1796 & 0.020 & 1024 \times 256 & 1.587 & 0.00146 & \text { Yes } & \text { Yes } \\ \text { G } & 0.03 & 2.30 & 76.7 & 2997 & 0.015 & 2048 \times 512 & 1.587 & 0.00088 & \text { Yes } & \text { Yes } \\ \text { H } & 0.015 & 2.46 & 164.0 & 5560 & 0.010 & 4096 \times 512 & 1.587 & 0.0005 & \text { Yes } & \text { Yes } \\ \text { I } & 0.02 & 0.98 & 49.0 & 269 & 0.026 & 512 \times 128 & 1.19 & 0.004 & \text { Yes } & \text { No } \\ \text { J } & 0.02 & 1.56 & 78.0 & 565 & 0.026 & 1024 \times 256 & 1.351 & 0.00276 & \text { Yes } & \text { No } \\ \text { K } & 0.01 & 0.95 & 95.0 & 1092 & 0.010 & 1024 \times 256 & 1.09 & 0.001 & \text { Yes } & \text { No } \\ \text { L } & 0.083 & 1.00 & 12.0 & 255 & 0.032 & 512 \times 128 & 1.19 & 0.004 & \text { No } & \text { Yes } \\ \text { M } & 0.12 & 1.90 & 15.8 & 472 & 0.042 & 512 \times 128 & 1.492 & 0.0044 & \text { No } & \text { Yes } \\ \text { N } & 0.04 & 1.10 & 27.5 & 1171 & 0.014 & 1024 \times 256 & 1.19 & 0.00094 & \text { No } & \text { Yes }\end{array}$

TABLE 1. Summary of all the runs discussed in the paper. The table lists the values of the sound Larmor radius $\rho_{s}$, length of the current sheet $L$, their ratio $L / \rho_{s}$, Lundquist number $S$, the inner boundary layer width $\delta_{\text {in }}$ (using (2.14)), the number of grid points employed $N_{x} \times N_{y}$, the length of domain in the $y$-direction, $L_{y}$, resistivity $\eta$ and answers whether both constraints of the semi-collisional regime are satisfied or not for a given run.

In the case of the three black inverted triangles shown in the figure 2, corresponding to Runs $\mathrm{L}, \mathrm{M}$ and $\mathrm{N}$, the ion sound Larmor radius $\rho_{s}$ is set to be larger than the SP current sheet width $\left(\delta_{\mathrm{SP}}\right)$. Thus, when $X$-point collapse happens, these runs are affected by ion scale physics; unsurprisingly, the current sheets here do not follow the SP scaling. In summary, by the end of the collapse of the primary tearing mode and formation of the current sheet, the blue starred runs (Runs A to $H$ ) are in the semi-collisional regime $\left(\delta_{\mathrm{SP}}>\rho_{s}>\delta_{\text {in }}\right)$, the red diamond runs (Runs I, J, K) in the 


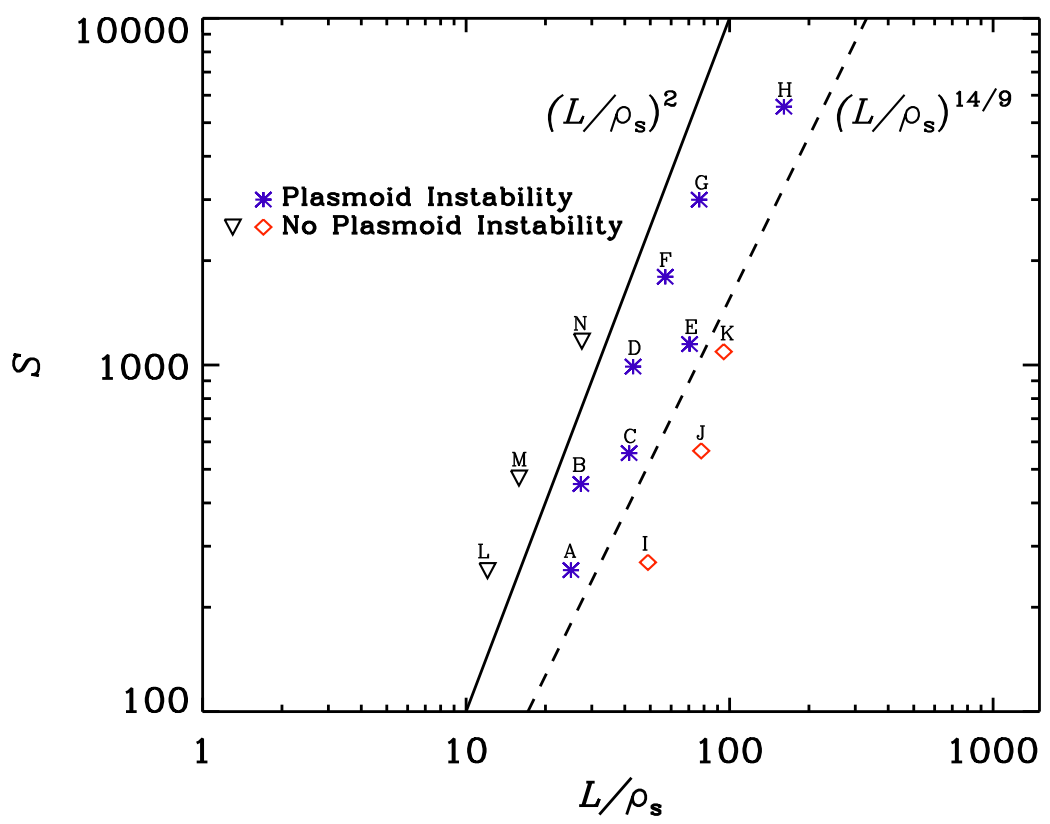

FIGURE 3. Reconnection phase diagram showing the semi-collisional regime marked out by the upper bound $\left(\delta_{\mathrm{SP}}>\rho_{s}\right.$; solid line) and lower bound $\left(\rho_{s}>\delta_{\text {in }}\right.$; dashed line) as prescribed by (2.15). Blue stars denote simulations which show the plasmoid instability; red diamonds and black inverted triangles correspond to simulations which do not. The parameters (and other details) of each run can be looked up in table 1.

MHD regime $\left(\delta_{\mathrm{SP}}>\delta_{\text {in }}>\rho_{s}\right)$ and the black inverted triangles (Runs L, M and N) in a weakly collisional regime where a Sweet-Parker sheet no longer forms $\left(\rho_{s}>\delta_{\mathrm{SP}}>\delta_{\text {in }}\right)$.

Next, we show whether or not the plasmoid instability is observed for a given run on the $S-L / \rho_{s}$ parameter space in figure 3. The two groups of runs represented by blue stars and red diamonds, which form SP current sheets, have $\rho_{s}<\delta_{\mathrm{SP}}$ (the symbols here match those in figure 2). Furthermore, in the case of the blue starred runs, upon the formation of the current sheet, the system becomes sensitive to the presence of $\rho_{s}$, as this kinetic scale is now larger than the semi-collisional inner boundary layer $\delta_{\text {in }}$ (corresponding to the newly formed SP layer) (see (2.8)). As a result, the plasmoid instability can arise, and indeed it does, as seen in Runs A to $\mathrm{H}$ (blue stars). On the other hand, in the case of Runs I, J, K (red diamonds), $\rho_{s}$ is not only smaller that $\delta_{\mathrm{SP}}$, but is also smaller that $\delta_{\text {in }}$ and thus no plasmoids arise in these runs. The condition of $\rho_{s}<\delta_{\text {in }}$ implies the absence of the plasmoid instability simply because the system continues to be in MHD regime and $S$ is much below the critical value of $\sim 10^{4}$ required in MHD.

The Runs A to $\mathrm{H}$ which do result in plasmoid instability are within the two theoretical bounds of the semi-collisional regime marked by solid and dashed black lines (2.15). Runs I, J and $\mathrm{K}$ (where $\rho_{s}<\delta_{\text {in }}$ ) below the lower bound match roughly in $S$ with Runs A, C and E respectively; Runs L, M, N (where $\rho_{s}>\delta_{\mathrm{SP}}$ ) above the upper bound, were performed to match in $S$ roughly with Runs A, B and E respectively. We find that neither of these two sets of runs yield the plasmoid instability. We conclude, therefore, that the theoretically prescribed bounds demarcating the semi-collisional regime are remarkably robust within the range of Lundquist numbers and Larmor radii that we have explored. 
(a)

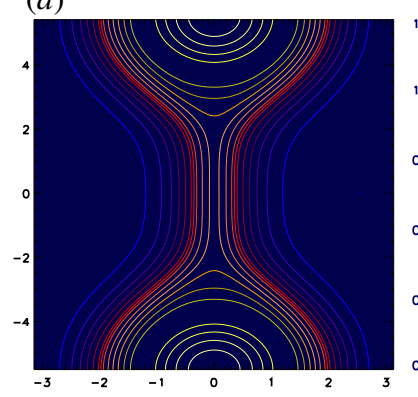

$(d)$

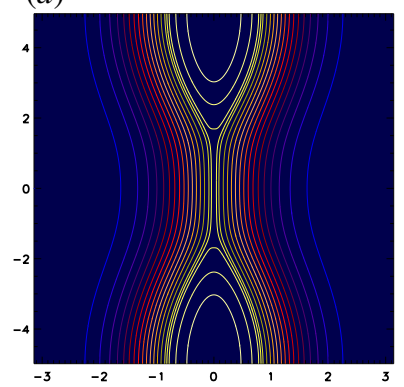

(b)

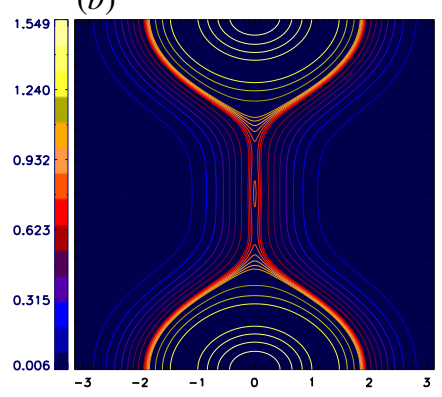

(e)

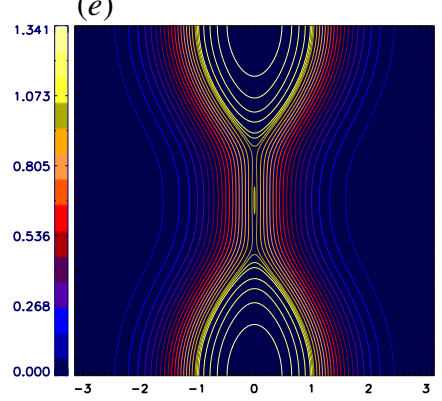

(c)

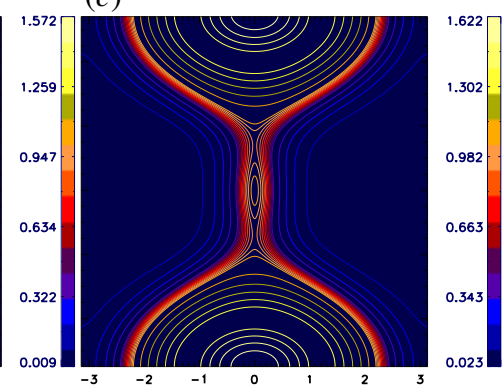

$(f)$

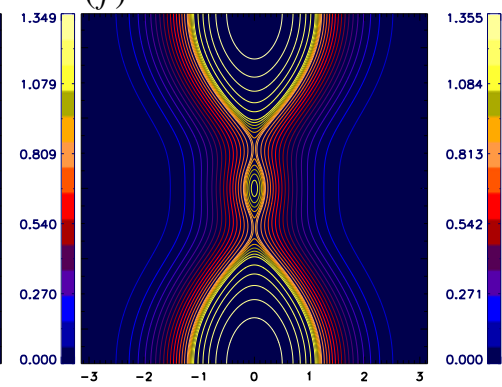

Figure 4. Contour plots of $A_{\|}$for Run A $(a-c)$ with lowest $S=256$ (and $L / \rho_{s}=25$ ), and Run $\mathrm{H}(d-f)$ with highest $S=5560$ (and $L / \rho_{s}=164$ ) at three different times: $(a, d)$, just before the plasmoid forms; $(b, e)$, in the early stages of plasmoid formation; and $(c, f)$, when the plasmoid is in its nonlinear stage.

Further insight can be gained by visually analysing these runs. In particular it is of interest to visually compare Runs $\mathrm{A}$ and $\mathrm{H}$, corresponding, respectively, to the lowest $(S=256)$ and highest $(S=5560)$ values of the Lundquist number at which we have observed the plasmoid instability. Figure 4 shows contour plots of $A_{\|}$at three different times. The two leftmost panels $(a, d)$ depict the system just before the plasmoid formation. The middle panels show the early stages of the plasmoid development; and the rightmost panels show the plasmoid well into the nonlinear stage. In both cases, note that in its early stage $(b, e)$ the $y$-extent of the plasmoid (roughly its linear wavelength) is much smaller than the length of the current sheet (to be discussed below). Also, due to the highly symmetric configuration of the magnetic field (and the intrinsic symmetry germane to the pseudo-spectral method that we employ), the plasmoid is stuck to the middle of the sheet. In a less constrained situation, we expect that this plasmoid would be ejected upwards or downwards, and subsequent plasmoids to be seeded until the system approaches saturation. It can be seen clearly that the gradient of $A_{\|}$in the current sheet is larger in Run $\mathrm{H}$ compared to Run A, as expected given the order of magnitude difference in the values of their Lundquist numbers. Thus a curiosity is that even in the run with highest $S$, only a single plasmoid forms. We will address this concern at a later point.

Another interesting comparison is between Runs E $\left(S=1149, L / \rho_{s}=70.5\right.$, blue star) and $\mathrm{K}\left(S=1092, L / \rho_{s}=95\right.$, red diamond). As seen in figure 5 , Run $\mathrm{E}$ shows a similar time evolution to that displayed by the runs in figure 4 , with the current sheet becoming unstable to plasmoid formation. In Run K, on the other hand, the collapsed current sheet never goes unstable, and the primary tearing mode just proceeds to 
(a)

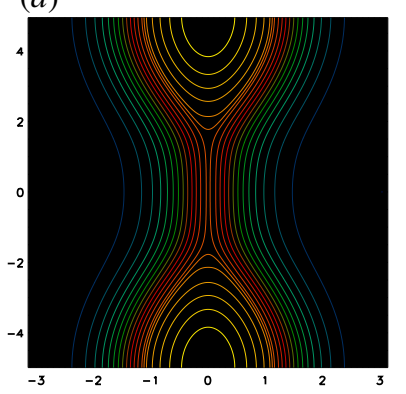

(d)

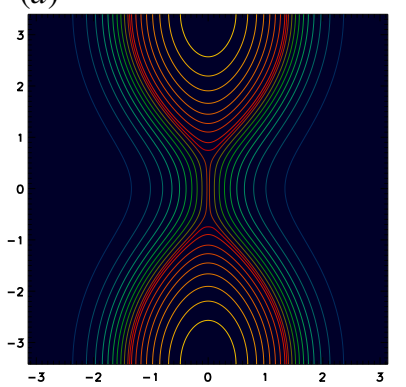

(b)

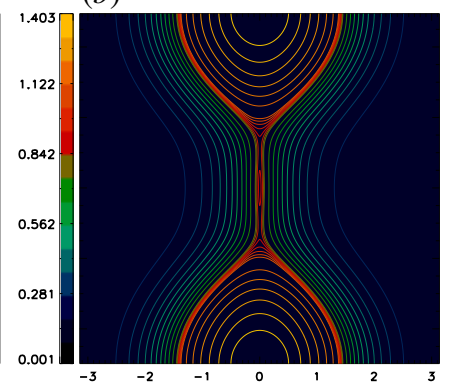

(e)

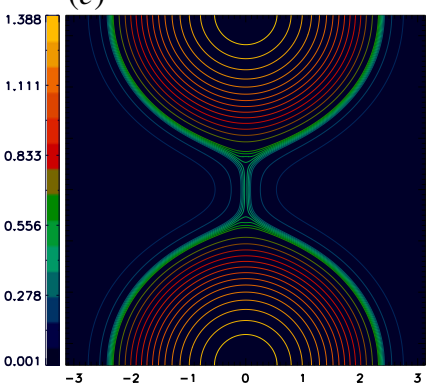

(c)

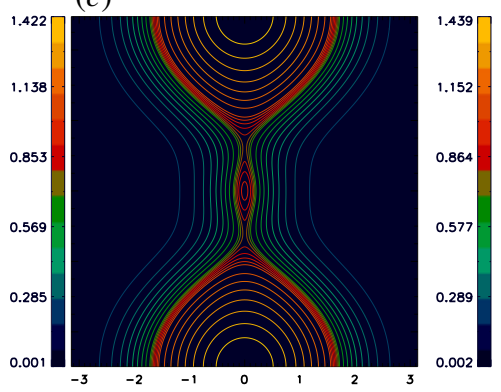

$(f)$

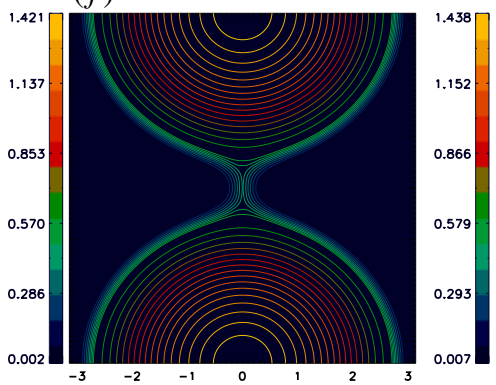

FIgURE 5. Contour plots of $A_{\|}$for Run E in the semi-collisional regime $(a-c)$, with $S=$ 1149 and $L / \rho_{s}=70.5$; and for Run K in the MHD regime $(d-f)$, with $S=1092$ and $L / \rho_{s}=95$, at three consecutive times from left to right.

(a)

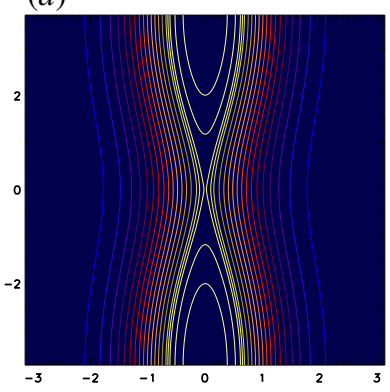

(b)

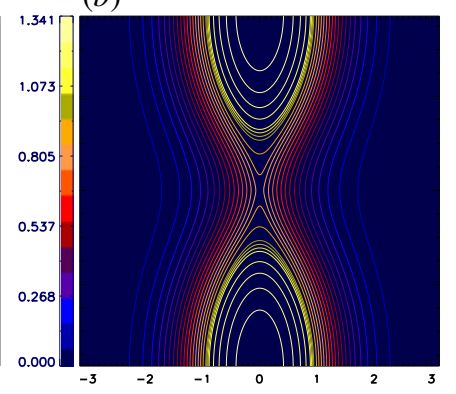

(c)

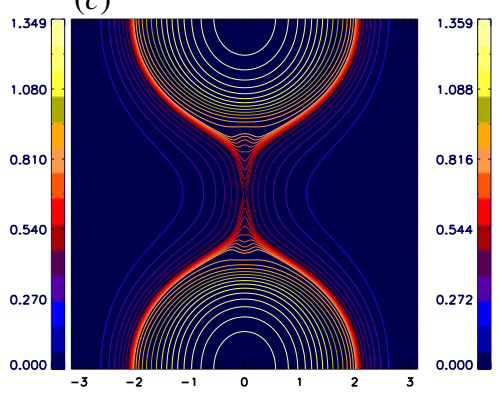

FIgURE 6. Contour plots of $A_{\|}$for Run N (in the weakly collisional regime), with $S=$ 1171 and $L / \rho_{s}=27.5$, at three consecutive times from $(a)$ to $(c)$.

saturation. This is rather remarkable given how close in the phase space outlined by $S$ and $L / \rho_{s}$ these two runs are (see figure 3).

In figure 6, we also show a run from the weakly collisional regime, with the parameters $S=1171$ and $L / \rho_{s}=27.5$ (Run $\mathrm{N}$ ). There is no plasmoid formation as expected. It is interesting to note that the current density forms an $X$-point, as seen from figure $6(a)$, which is consistent with what is expected in such a regime. The $X$-point later transitions to a double-structured current sheet, which retains a sharp peak at its centre.

It is noteworthy that all of the runs in this regime are at Lundquist numbers much lower than the MHD critical value of order $\sim 10^{4}$. The lowest $S$ at which a simulation 


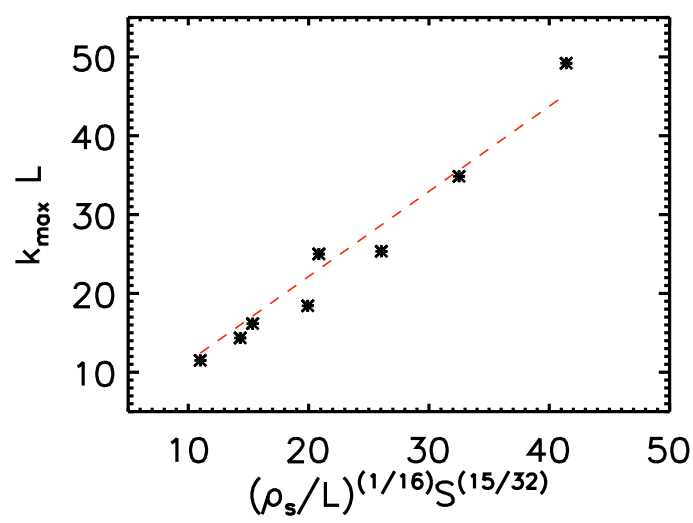

FIGURE 7. Wavenumber measured from the width of the arising plasmoid in Runs A-G, plotted against the theoretical prediction (2.13).

(Run A) obtains the plasmoid instability is 250. As one decreases the value of $S$ even further we find in our simulations that width of the primary island becomes as large as the simulation domain before any plasmoid is observed. In a less constrained system it is possible that the plasmoid instability remains active at even lower values of $S$.

The linear regime of the plasmoid instability is very short lived: it ends once the plasmoid becomes larger than the inner boundary layer. Although this layer is resolved in all our simulations, the number of grid points in the inner layer is not sufficiently large to be able to trace a long enough evolution in the linear regime. Thus, linear growth rate measurements cannot reliably be obtained. However, the wavenumber is a straightforward quantity to measure. An intriguing observation is that only one single plasmoid arises in the Runs $\mathrm{A}-\mathrm{H}$. These runs range over a decade in Lundquist number and thus the expected number of plasmoids varies by a factor of $\gtrsim 3$ from the run with lowest $S$ to the run with highest $S$, according to (2.13). That is not what we obtain, suggesting disagreement with the linear predictions. This leads one to wonder why it is that the lower bound, $S>\left(L / \rho_{s}\right)^{14 / 9}$, is validated so well (figure 3), given that its validity relies on the scalings for $k_{\max }$ (and $\gamma_{\max }$ ) being correct.

To address this issue, we proceed as follows. For each run, instead of counting the number of plasmoids to estimate the wavenumber, we compute it from the measurement of the $y$-extent of the plasmoid (full width at half-maximum of the island in $A_{\|}$) at the earliest possible stages of its appearance. We find that this measurement of the wavenumber does follow the theoretical scaling rather well, as shown in figure 7. We suspect that the explanation for this result lies in the effects of spatial inhomogeneity in the direction along the sheet, as well as flows and reconnected component of the magnetic field. None of these effects is negligible at these low values of the Lundquist number, but they are all neglected in the theoretical derivation. This argument is strengthened further by the results shown in the Appendix. The important observation, however, is that the plasmoid that does form has the correct wavenumber as predicted by linear theory.

\section{Discussions and conclusions}

In this paper, we have investigated, analytically and numerically, the semi-collisional regime of the plasmoid instability - an extension to a Sweet-Parker sheet of the 
semi-collisional tearing mode (Drake \& Lee 1977; Cowley, Kulsrud \& Hahm 1986; Zocco \& Schekochihin 2011). We employ a reduced kinetic formalism obtainable from the gyrokinetic formalism via asymptotic expansion in low plasma $\beta$ (Zocco \& Schekochihin 2011). The simulations are carried out with the code Viriato (Loureiro et al. 2016). This regime is analytically predicted to occupy a significant sliver of the reconnection phase diagram defined by a lower bound $S>\left(L / \rho_{S}\right)^{14 / 9}$ and an upper bound $S<\left(L / \rho_{s}\right)^{2}$. Our numerical simulations show that these bounds are remarkably robust: runs which fall within these two bounds yield the plasmoid instability, and vice versa. The instability arises at much lower values of the Lundquist numbers than the MHD analogue; as low as $S \sim 250$. We are limited in our exploration of even lower values of $S$ by our simulation set-up. Thus, we do not rule out the formation of plasmoids for $S<250$ in less constrained systems; indeed, we speculate that this regime could also potentially explain formation of plasmoids in recent reconnection experiments (Jara-Almonte et al. 2016; Hare et al. 2017a,b, 2018). In Magpie, for example, we find from table I in Hare et al. (2018) that $S \approx 120$ and $L / d_{i} \approx 18$. If we were to consider the relevant kinetic scale to be $d_{i}$ instead of $\rho_{s}$, then the value of these parameters $\left(S\right.$ and $\left.L / d_{i}\right)$ satisfy the condition for the semi-collisional regime and fall within its bounds. Note that in the collisionless (or weakly collisional) regime, plasmoids arise only when $L / \rho_{s}$ is larger than a value of about $\sim 50$ (an empirical value seen in simulations) (Ji \& Daughton 2011), whereas the semi-collisional regime seems to offer no hard lower bound. Further, with the validation of the existence of this regime, the reconnection phase diagram (figure 1 in Ji \& Daughton 2011) would be modified. The lower bound of this regime, governed by either $S=\left(L / \rho_{s}\right)^{14 / 9}$ or $S=\left(L / \rho_{s}\right)^{8 / 5}$ would now cut across the two lines - one line representing the critical Lundquist number for MHD plasmoid instability $\left(S=S_{c}\right)$ and then a second line representing the lower bound of the multiple $X$-line hybrid regime $\left(S=L \sqrt{S_{c}} / 2 \rho_{s}\right)$.

The numerical experiments reported here are limited in that the amount of flux to reconnect is finite, and the simulation box is periodic. As such, (statistical) steady state reconnection cannot be attained, thereby preventing us from numerically answering the important question of what the reconnection rate is in the semi-collisional plasmoid regime. However, theoretically we may expect the following. In the phase-space diagram of figure 3 , assume that the initial system, with a certain $S, L$ and $\rho_{s}$, is in the semi-collisional regime. As the plasmoid instability unfolds, we expect that smaller, interplasmoid current sheets will arise. These will necessarily have a smaller length, $L^{\prime} \sim L / N$, where $N$ is the number of primary plasmoids. Each of these interplasmoid current sheets now defines a reconnecting site which can be located in the reconnection phase diagram. Since $\rho_{s}$ and $\eta$ are fixed, and assuming that $V_{A}$ is the same in these daughter sheets, the only parameter that has changed is the length, from $L$ to $L^{\prime}$. This means a diagonal displacement in the direction of smaller $L / \rho_{s}$ from the initial point in that diagram; the slope of that diagonal is 1 , because both axes are linearly proportional to $L$. If the new position in this diagram remains in the semi-collisional regime, each interplasmoid current sheet is still unstable to the semi-collisional plasmoid instability. The process then repeats (i.e. the plasmoid hierarchy unfolds further) until arriving at an interplasmoid current sheet which is now short enough to be outside of the semi-collisional bounds. Inevitably, therefore, this lands the system to the left of the $S \sim\left(L / \rho_{s}\right)^{2}$ bound, i.e. the collisionless regime, where the expected reconnection rate is $\sim 0.1 \tau_{A}^{-1}$. Defining $\lambda_{c}=\left(L_{c} / \rho_{s}\right)$ as an empirical scale separating the single from the multiple $X$-line collisionless regimes (numerically observed to be $\sim 50$ ), we conclude that the system finally lands in either the multiple, or single, $X$-line collisionless regime depending on whether it is initially 
above or below the diagonal line $S \sim \lambda_{c}\left(L / \rho_{s}\right)$, (which intersects the line $S \sim\left(L / \rho_{s}\right)^{2}$ at $\left.\left(L / \rho_{s}\right)=\lambda_{c}\right)$ in the reconnection phase space diagram.

Finally, let us outline some general arguments for the case when, unlike our simulations, the global Lundquist number of the system is so large that a SweetParker current sheet may not be able to form dynamically. Consider then a forming current sheet, and assume for simplicity that the characteristic time at which it is forming is Alfvénic, $\tau_{A}=L / V_{A}$. At any given moment of time, we parameterize the forming current sheet aspect ratio as $a / L=S^{-\alpha}$ (Pucci \& Velli 2014), where $a$ is the current sheet width, and $\alpha$ is a number such that $0<\alpha<1 / 2$, with $\alpha=1 / 2$ representing a Sweet-Parker sheet (which the system presumably never gets to).

Using (2.6) (for a Harris-type sheet) we see that the growth rate of the most unstable semi-collisional mode exceeds the current sheet formation rate (Alfvénic) when

$$
\gamma_{\max } \tau_{A} \sim\left(\rho_{s} / L\right)^{2 / 3} S^{-1 / 3+2 \alpha} \gtrsim 1 .
$$

This expression constrains the relationship between $S$ and $L / \rho_{s}$, for any value of $\alpha$, to attain Alfvénic growth at that value of $\alpha$. In addition, we must further require that this mode (the most unstable semi-collisional mode) is indeed in the semi-collisional regime, i.e. $\delta_{\text {in, } \max }^{S C}<\rho_{s}$. This leads to,

$$
\left(\rho_{s} / L\right)^{1 / 9} S^{-(2 \alpha / 3)-2 / 9}<\rho_{s} / L .
$$

These two expressions intersect when $\alpha=1 / 3$. That is, if $\alpha<1 / 3$, the relationship that $S$ and $L / \rho_{s}$ must satisfy to yield Alfvénic growth is given by (6.1); if instead $\alpha>1 / 3$, then it is sufficient to satisfy (6.2) to attain Alfvénic growth. ${ }^{6}$

A detailed analysis of all different possibilities that are encountered as $\alpha$ increases is beyond the scope of this paper and will be left to future work. Generally, for $\alpha<1 / 3$, the condition for the fastest semi-collisional mode to become faster than Alfvénic becomes progressively less demanding on $S$. Consider the particularly interesting case where $\alpha$ reaches the value of $1 / 3$. At this value, we must have $S>\left(L / \rho_{s}\right)^{2}$ for the semi-collisional mode to both exist and be super-Alfvénic. Remarkably, $\alpha=1 / 3$ is the value at which the fastest growing MHD mode becomes Alfvénic (Pucci \& Velli 2014). The condition for that mode to indeed be in the MHD regime is, unsurprisingly, just the reverse of the above, $S<\left(L / \rho_{s}\right)^{2}$. So, in this case $(\alpha=1 / 3)$, the outcome is particularly simple: if $S>\left(L / \rho_{s}\right)^{2}$ the forming sheet would be disrupted by a semicollisional mode, and one might expect the reconnection rate to ensue to be $0.1 \tau_{A}^{-1}$ as discussed above; if, instead $S<\left(L / \rho_{S}\right)^{2}$ then the sheet would be disrupted by an MHD mode, and the reconnection rate would presumably be $S_{c}^{-1 / 2} \tau_{A}^{-1} \sim 0.01 \tau_{A}^{-1}$ (unless a transition to the semi-collisional regime occurs during the plasmoid cascade - see footnote 4 on page 5). For example, in the solar corona, where $S \sim 10^{13}$ and $L / \rho_{s} \sim$ $10^{7}$, we see that the MHD mode would win.

\section{Acknowledgements}

We thank E. Tolman and R. White for helpful discussions. This work was supported by the NSF-DOE Partnership in Basic Plasma Science and Engineering, Award no. DE-SC0016215. The simulations presented in this paper were performed on the MITPSFC partition of the Engaging cluster at the MGHPCC facility (www.mghpcc.org) which was funded by DoE grant no. DE-FG02-91-ER54109. We also acknowledge the usage of Stampede cluster in Texas under the allocation TG-PHY140041.

\footnotetext{
${ }^{6}$ If instead one considers the case of a sinusoidal-like current sheet, equation (6.1) is replaced by $\gamma_{\max } \tau_{A} \sim$ $\left(\rho_{S} / L\right)^{5 / 8} S^{-1 / 4+(15 \alpha / 8)} \gtrsim 1$; and $(6.2)$ is replaced by $\left(\rho_{S} / L\right)^{1 / 8} S^{-(5 \alpha / 8)-1 / 4}<\rho_{S} / L$. Their intersection now takes place at $\alpha=3 / 10$. All the same arguments apply to this case.
} 

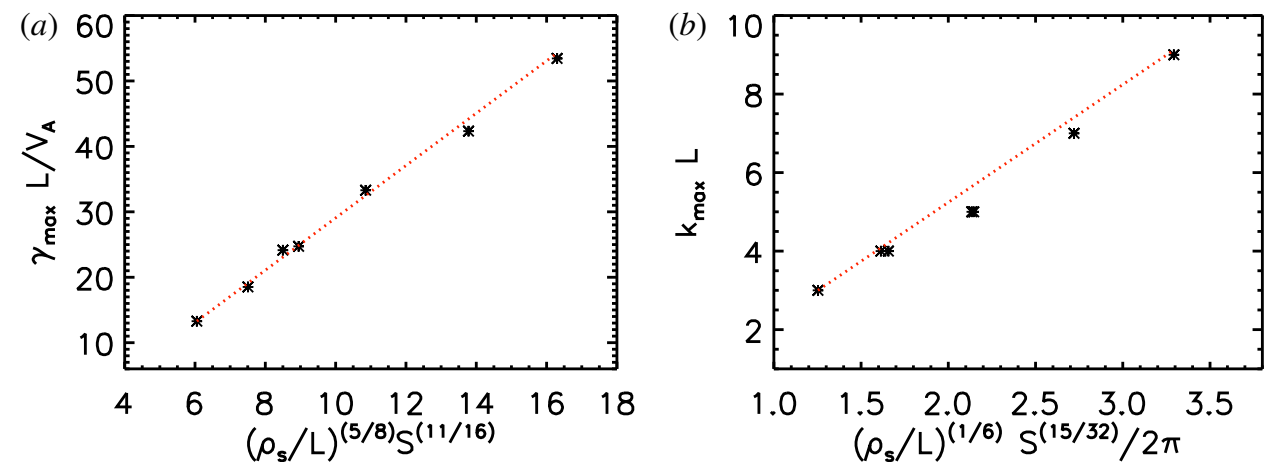

FIGURE 8. Growth rate $(a)$ and wavenumber $(b)$ of the most unstable semi-collisional plasmoid mode in a prescribed Sweet-Parker-like sheet, plotted against the theoretical predictions, equations (2.12) and (2.13).

\section{Appendix. Semi-collisional tearing mode scalings in an SP sheet}

To confirm the validity of the analytical derivation of $\S 2$, and the ability of the Viriato code to recover the scalings predicted there, we have performed a set of simulations whose key difference from those reported in the main text lies in the fact that the thickness of initial magnetic profile is now the Sweet-Parker width, $L_{y} S^{-1 / 2}$, as is assumed throughout $\S 2$. This initial configuration is not an exact Sweet-Parker sheet because it lacks both the appropriate flows, and the reconnected component of the magnetic field; these are also simplifications adopted in the theoretical derivation, and which can be shown analytically to be justifiable (Loureiro et al. 2007, 2013).

All possible modes are seeded by the introduction of a small amplitude random perturbation at $t=0$. After an initial transient, we observe the exponential growth of a single mode - the most unstable perturbation. The growth rate and wavenumber (determined by counting the number of arising plasmoids) of this mode are plotted in figure 8. Excellent agreement with the theoretical scalings of (2.12) and (2.13) is observed.

\section{REFERENCES}

Batlrud, S. D., Bhattacharjee, A., Huang, Y.-M. \& Germaschewski, K. 2011 Hall magnetohydrodynamic reconnection in the plasmoid unstable regime. Phys. Plasmas 18 (9), 092108 .

BATY, H. 2014 Effect of plasma- $\beta$ on the onset of plasmoid instability in Sweet-Parker current sheets. J. Plasma Phys. 80, 655-665.

Bhattacharjee, A., Huang, Y.-M., Yang, H. \& Rogers, B. 2009 Fast reconnection in highLundquist-number plasmas due to the plasmoid instability. Phys. Plasmas 16 (11), 112102.

BISKAMP, D. 1986 Magnetic reconnection via current sheets. Phys. Fluids 29, 1520-1531.

BISKAMP, D. 2000 Magnetic Reconnection in Plasmas. Cambridge University Press.

Burch, J. L., Torbert, R. B., Phan, T. D., Chen, L.-J., Moore, T. E., Ergun, R. E., Eastwood, J. P., Gershman, D. J., Cassak, P. A., Argall, M. R. et al. 2016 Electronscale measurements of magnetic reconnection in space. Science 352, aaf2939.

CASSAK, P. A., Shay, M. A. \& Drake, J. F. 2009 Scaling of Sweet-Parker reconnection with secondary islands. Phys. Plasmas 16 (12), 120702.

Cerri, S. S. \& CAlifano, F. 2017 Reconnection and small-scale fields in 2D-3V hybrid-kinetic driven turbulence simulations. New J. Phys. 19 (2), 025007. 
Cerutti, B., Uzdensky, D. A. \& Begelman, M. C. 2012 Extreme particle acceleration in magnetic reconnection layers: application to the gamma-ray flares in the crab nebula. Astrophys. J. 746, 148.

Cerutti, B., Werner, G. R., Uzdensky, D. A. \& Begelman, M. C. 2013 Simulations of particle acceleration beyond the classical synchrotron burnoff limit in magnetic reconnection: an explanation of the crab flares. Astrophys. J. 770, 147.

Comisso, L., Lingam, M., Huang, Y.-M. \& Bhattacharjee, A. 2016 General theory of the plasmoid instability. Phys. Plasmas 23 (10), 100702.

Cowley, S. C., Kulsrud, R. M. \& Hahm, T. S. 1986 Linear stability of tearing modes. Phys. Fluids 29, 3230-3244.

DAUGhTON, W. \& ROYTERSHTEYN, V. 2012 Emerging parameter space map of magnetic reconnection in collisional and kinetic regimes. Space Sci. Rev. 172, 271-282.

Dibraccio, G. A., Slavin, J. A., Imber, S. M., Gershman, D. J., Raines, J. M., Jackman, C. M., Botrdsen, S. A., Anderson, B. J., Korth, H., Zurbuchen, T. H. et al. 2015 MESSENGER observations of flux ropes in Mercury's magnetotail. Planet. Space Sci. 115, $77-89$.

Dorfman, S., Ji, H., Yamada, M., Yoo, J., Lawrence, E., Myers, C. \& Tharp, T. D. 2013 Three-dimensional, impulsive magnetic reconnection in a laboratory plasma. Geophys. Rev. Lett. 40, 233-238.

Drake, J. F. \& Lee, Y. C. 1977 Kinetic theory of tearing instabilities. Phys. Fluids 20, 1341-1353.

Drake, J. F., Swisdak, M., Che, H. \& Shay, M. A. 2006 Electron acceleration from contracting magnetic islands during reconnection. Nature 443, 553-556.

Dungey, J. W. 1961 Interplanetary magnetic field and the auroral zones. Phys. Rev. Lett. 6, 47-48.

Forest, C. B., Flanagan, K., Brookhart, M. \& Clark, M. 2015 The Wisconsin plasma astrophysics laboratory. J. Plasma Phys. 81, 345810501.

Fox, W., Bhattacharjee, A. \& Germaschewski, K. 2012 Magnetic reconnection in high-energydensity laser-produced plasmasa). Phys. Plasmas 19 (5), 056309.

Frieman, E. A. \& Chen, L. 1982 Nonlinear gyrokinetic equations for low-frequency electromagnetic waves in general plasma equilibria. Phys. Fluids 25, 502-508.

Furth, H. P., Killeen, J. \& Rosenbluth, M. N. 1963 Finite-resistivity instabilities of a sheet pinch. Phys. Fluids 6, 459-484.

Giannios, D., Uzdensky, D. A. \& Begelman, M. C. 2009 Fast TeV variability in blazars: jets in a jet. Mon. Not. R. Astron. Soc. 395, L29-L33.

Guo, F., LiU, Y.-H., Daughton, W. \& LI, H. 2015 Particle acceleration and plasma dynamics during magnetic reconnection in the magnetically dominated regime. Astrophys. J. 806, 167.

HARE, J. D. 2017 High energy density magnetic reconnection experiments in colliding carbon plasma flows. PhD thesis, Imperial College London.

Hare, J. D., Lebedev, S. V., Suttle, L. G., Loureiro, N. F., Ciardi, A., Burdiak, G. C., Chittenden, J. P., Clayson, T., Eardley, S. J., Garcia, C. et al. 2017 a Formation and structure of a current sheet in pulsed-power driven magnetic reconnection experiments. Phys. Plasmas 24 (10), 102703.

Hare, J. D., Suttle, L., Lebedev, S. V., Loureiro, N. F., Ciardi, A., Burdiak, G. C., Chittenden, J. P., Clayson, T., Garcia, C., Niasse, N. et al. 2017b Anomalous heating and plasmoid formation in a driven magnetic reconnection experiment. Phys. Rev. Lett. 118 (8), 085001.

Hare, J. D., Suttle, L. G., Lebedev, S. V., Loureiro, N. F., Ciardi, A., Chittenden, J. P., Clayson, T., Eardley, S. J., Garcia, C., Halliday, J. W. D. et al. 2018 An experimental platform for pulsed-power driven magnetic reconnection. Phys. Plasmas 25 (5), 055703.

HARRIS, E. G. 1962 On a plasma sheath separating regions of oppositely directed magnetic field. Il Nuovo Cimento 23, 115-121.

Hastie, R. J. 1997 Sawtooth instability in tokamak plasmas. Astrophys. Space Sci. 256, 177-204. 
Howes, G. G., Cowley, S. C., Dorland, W., Hammett, G. W., Quataert, E. \& Schekochinin, A. A. 2006 Astrophysical gyrokinetics: basic equations and linear theory. Astrophys. J. 651, 590-614.

HuAng, Y.-M. \& BhattacharJee, A. 2010 Scaling laws of resistive magnetohydrodynamic reconnection in the high-Lundquist-number, plasmoid-unstable regime. Phys. Plasmas 17 (6), 062104.

Jackman, C. M., Slavin, J. A. \& Cowley, S. W. H. 2011 Cassini observations of plasmoid structure and dynamics: implications for the role of magnetic reconnection in magnetospheric circulation at Saturn. J. Geophys. Res. Space Phys. 116, A10212.

Jara-Almonte, J., Ji, H., Yamada, M., Yoo, J. \& FoX, W. 2016 Laboratory observation of resistive electron tearing in a two-fluid reconnecting current sheet. Phys. Rev. Lett. 117 (9), 095001.

Ji, H., Bhattacharjee, A., Prager, S., Daughton, W., Bale, S. D., Carter, T., Crocker, N., DRAKe, J., EgedAl, J., SARFF, J. et al. 2015 FLARE (facility for laboratory reconnection experiments): a major next-step for laboratory studies of magnetic reconnection. In AAS/AGU Triennial Earth-Sun Summit, AAS/AGU Triennial Earth-Sun Summit, vol. 1, p. 104.05.

Ji, H. \& DAughton, W. 2011 Phase diagram for magnetic reconnection in heliophysical, astrophysical, and laboratory plasmas. Phys. Plasmas 18 (11), 111207.

Kagan, D., Sironi, L., Cerutti, B. \& Giannios, D. 2015 Relativistic magnetic reconnection in pair plasmas and its astrophysical applications. Space Sci. Rev. 191 (1), 545-573.

Lapenta, G. 2008 Self-feeding turbulent magnetic reconnection on macroscopic scales. Phys. Rev. Lett. 100 (23), 235001.

Lenard, A. \& Bernstein, I. B. 1958 Plasma oscillations with diffusion in velocity space. Phys. Rev. 112, 1456-1459.

Liu, W., Chen, Q. \& Petrosian, V. 2013 Plasmoid ejections and loop contractions in an eruptive M7.7 solar flare: evidence of particle acceleration and heating in magnetic reconnection outflows. Astrophys. J. 767, 168.

LOUREIRo, N. F. \& BoldyREV, S. 2017 Role of magnetic reconnection in magnetohydrodynamic turbulence. Phys. Rev. Lett. 118 (24), 245101.

Loureiro, N. F., Cowley, S. C., Dorland, W. D., Haines, M. G. \& Schekochinin, A. A. $2005 X$-point collapse and saturation in the nonlinear tearing mode reconnection. Phys. Rev. Lett. 95 (23), 235003.

Loureiro, N. F., Dorland, W., Fazendeiro, L., Kanekar, A., Mallet, A., Vilelas, M. S. \& ZocCO, A. 2016 Viriato: a Fourier-Hermite spectral code for strongly magnetized fluid-kinetic plasma dynamics. Comput. Phys. Commun. 206, 45-63.

Loureiro, N. F., Samtaney, R., Schekochinin, A. A. \& Uzdensky, D. A. 2012 Magnetic reconnection and stochastic plasmoid chains in high-Lundquist-number plasmas. Phys. Plasmas 19 (4), 042303.

Loureiro, N. F., Schekochinin, A. A. \& Cowley, S. C. 2007 Instability of current sheets and formation of plasmoid chains. Phys. Plasmas 14 (10), 100703.

Loureiro, N. F., Schekochinin, A. A. \& Uzdensky, D. A. 2013 Plasmoid and Kelvin-Helmholtz instabilities in Sweet-Parker current sheets. Phys. Rev. E 87 (1), 013102.

Loureiro, N. F. \& UzDEnSKy, D. A. 2016 Magnetic reconnection: from the Sweet-Parker model to stochastic plasmoid chains. Plasma Phys. Control. Fusion 58 (1), 014021.

Mallet, A., Schekochinin, A. A. \& Chandran, B. D. G. 2017 Disruption of sheet-like structures in Alfvenic turbulence by magnetic reconnection. Mon. Not. R. Astron. Soc. 468, $4862-4871$.

Matthaeus, W. H. \& Lamkin, S. L. 1986 Turbulent magnetic reconnection. Phys. Fluids 29, 2513-2534.

Milligan, R. O., McAteer, R. T. J., Dennis, B. R. \& Young, C. A. 2010 Evidence of a plasmoid-looptop interaction and magnetic inflows during a solar flare/coronal mass ejection eruptive event. Astrophys. J. 713, 1292-1300. 
Moldwin, M. B. \& Hughes, W. J. 1992 On the formation and evolution of plasmoids - a survey of ISEE 3 geotail data. J. Geophys. Res. 97, 19.

Nishizuka, N., TAKasaki, H., Asai, A. \& Shibata, K. 2010 Multiple plasmoid ejections and associated hard X-ray bursts in the 2000 November 24 flare. Astrophys. J. 711, 1062-1072.

NumAtA, R. \& Loureiro, N. F. 2015 Ion and electron heating during magnetic reconnection in weakly collisional plasmas. J. Plasma Phys. 81 (2), 305810201.

Oka, M., Phan, T.-D., Krucker, S., Fujimoto, M. \& Shinohara, I. 2010 Electron acceleration by multi-island coalescence. Astrophys. J. 714 (1), 915.

Olson, J., Egedal, J., Greess, S., Myers, R., Clark, M., Endrizzi, D., Flanagan, K., Milhone, J., Peterson, E., Wallace, J. et al. 2016 Experimental demonstration of the collisionless plasmoid instability below the ion kinetic scale during magnetic reconnection. Phys. Rev. Lett. 116 (25), 255001.

PARKER, E. N. 1957 Sweet's mechanism for merging magnetic fields in conducting fluids. J. Geophys. Res. 62, 509-520.

Pegoraro, F. \& SCHeP, T. J. 1986 Theory of resistive modes in the ballooning representation. Plasma Phys. Control. Fusion 28, 647-667.

Pucci, F. \& Velli, M. 2014 Reconnection of quasi-singular current sheets: the 'ideal' tearing mode. Astrophys. J. Lett. 780, L19.

Samtaney, R., Loureiro, N. F., Uzdensky, D. A., Schekochinin, A. A. \& Cowley, S. C. 2009 Formation of plasmoid chains in magnetic reconnection. Phys. Rev. Lett. 103 (10), 105004.

Servidio, S., Matthaeus, W. H., Shay, M. A., Cassak, P. A. \& Dmitruk, P. 2009 Magnetic reconnection in two-dimensional magnetohydrodynamic turbulence. Phys. Rev. Lett. 102 (11), 115003.

Sharma, R., Mitra, D. \& Oberoi, D. 2017 On the energization of charged particles by fast magnetic reconnection. Mon. Not. R. Astron. Soc. 470, 723-731.

Shibata, K. \& Magara, T. 2011 Solar flares: magnetohydrodynamic processes. Living Rev. Sol. Phys. 8, 6.

Sironi, L. \& SpITKOVSKy, A. 2014 Relativistic reconnection: an efficient source of non-thermal particles. Astrophys. J. Lett. 783, L21.

SwEET, P. A. 1958 The neutral point theory of solar flares. In Electromagnetic Phenomena in Cosmical Physics (ed. B. Lehnert), IAU Symposium, vol. 6, p. 123.

Tajima, T. \& Shibata, K. 2002 Plasma Astrophysics. Perseus.

Tolman, E. A., Loureiro, N. F. \& Uzdensky, D. A. 2018 Development of tearing instability in a current sheet forming by sheared incompressible flow. J. Plasma Phys. 84 (1), 905840115.

UZDENSKY, D. A. 2011 Magnetic reconnection in extreme astrophysical environments. Space Sci. Rev. 160, 45-71.

Uzdensky, D. A. \& Loureiro, N. F. 2016 Magnetic reconnection onset via disruption of a forming current sheet by the tearing instability. Phys. Rev. Lett. 116 (10), 105003.

Uzdensky, D. A., Loureiro, N. F. \& ScheKochihin, A. A. 2010 Fast magnetic reconnection in the plasmoid-dominated regime. Phys. Rev. Lett. 105 (23), 235002.

WAelbroeck, F. L. 1989 Current sheets and nonlinear growth of the $m=1$ kink-tearing mode. Phys. Fluids B 1, 2372-2380.

Werner, G. R. \& UzDEnsky, D. A. 2017 Nonthermal particle acceleration in 3D relativistic magnetic reconnection in pair plasma. Astrophys. J. Lett. 843, L27.

Werner, G. R., Uzdensky, D. A., Cerutti, B., Nalewajko, K. \& Begelman, M. C. 2016 The extent of power-law energy spectra in collisionless relativistic magnetic reconnection in pair plasmas. Astrophys. J. Lett. 816, L8.

White, R. L. \& Hazeltine, R. D. 2017 Analysis of the Hermite spectrum in plasma turbulence. Phys. Plasmas 24 (10), 102315.

Yamada, M., Ji, H., Hsu, S., Carter, T., Kulsrud, R., Bretz, N., Jobes, F., Ono, Y. \& Perkins, F. 1997 Study of driven magnetic reconnection in a laboratory plasma. Phys. Plasmas 4, 1936-1944. 
Zhang, Q. M. \& JI, H. S. 2014 Blobs in recurring extreme-ultraviolet jets. Astron. Astrophys. 567, A11.

Zhang, T. L., Lu, Q. M., Baumjohann, W., Russell, C. T., Fedorov, A., Barabash, S., Coates, A. J., Du, A. M., CAO, J. B., NaKamura, R. et al. 2012 Magnetic reconnection in the near venusian magnetotail. Science 336, 567.

Zhdankin, V., Uzdensky, D. A., Perez, J. C. \& Boldyrev, S. 2013 Statistical analysis of current sheets in three-dimensional magnetohydrodynamic turbulence. Astrophys. J. 771, 124.

Zhou, X., BÜChner, J., BÁrTA, M., GAN, W. \& LiU, S. 2015 Electron acceleration by cascading reconnection in the solar corona. I. Magnetic gradient and curvature drift effects. Astrophys. $J$. $815,6$.

Zocco, A., Loureiro, N. F., Dickinson, D., Numata, R. \& Roach, C. M. 2015 Kinetic microtearing modes and reconnecting modes in strongly magnetised slab plasmas. Plasma Phys. Control. Fusion 57 (6), 065008.

Zocco, A. \& Schekochinin, A. A. 2011 Reduced fluid-kinetic equations for low-frequency dynamics, magnetic reconnection, and electron heating in low-beta plasmas. Phys. Plasmas $\mathbf{1 8}$ (10), 102309.

Zong, Q.-G., Fritz, T. A., Pu, Z. Y., Fu, S. Y., Baker, D. N., Zhang, H., Lui, A. T., Vogiatzis, I., Glassmeier, K.-H., Korth, A. et al. 2004 Cluster observations of earthward flowing plasmoid in the tail. Geophys. Rev. Lett. 31, L18803.

Zweibel, E. G. \& YAmadA, M. 2009 Magnetic reconnection in astrophysical and laboratory plasmas. Annu. Rev. Astron. Astrophys. 47, 291-332. 